\title{
Optimal selection on two quantitative trait loci with linkage
}

\author{
Jack C.M. Dekkers ${ }^{\mathrm{a} *}$, Reena Chakraborty ${ }^{\mathrm{a}}$, \\ Laurence MOREAU ${ }^{\mathrm{b}}$ \\ ${ }^{\text {a }}$ Department of Animal Science, 225 Kildee Hall, \\ Iowa State University Ames, IA, 50011, USA \\ b INRA-UPS-INA PG, Station de génétique végétale, \\ Ferme du Moulon, 91190 Gif-sur-Yvette, France
}

(Received 5 February 2001; accepted 15 October 2001)

\begin{abstract}
A mathematical approach to optimize selection on multiple quantitative trait loci (QTL) and an estimate of residual polygenic effects was applied to selection on two linked or unlinked additive QTL. Strategies to maximize total or cumulative discounted response over ten generations were compared to standard QTL selection on the sum of breeding values for the QTL and an estimated breeding value for polygenes, and to phenotypic selection. Optimal selection resulted in greater response to selection than standard QTL or phenotypic selection. Tight linkage between the QTL (recombination rate 0.05 ) resulted in a slightly lower response for standard QTL and phenotypic selection but in a greater response for optimal selection. Optimal selection capitalized on linkage by emphasizing selection on favorable haplotypes. When the objective was to maximize total response after ten generations and QTL were unlinked, optimal selection increased QTL frequencies to fixation in a near linear manner. When starting frequencies were equal for the two QTL, equal emphasis was given to each QTL, regardless of the difference in effects of the QTL and regardless of the linkage, but the emphasis given to each of the two QTL was not additive. These results demonstrate the ability of optimal selection to capitalize on information on the complex genetic basis of quantitative traits that is forthcoming.
\end{abstract}

selection / marker-assisted selection / quantitative trait loci / optimization

\section{INTRODUCTION}

The advent of molecular genetics has opened opportunities to enhance selection for quantitative traits by incorporating information on the identified quantitative trait loci (QTL) or on the genetic markers that are linked to QTL in genetic improvement programs. In what follows, we will refer to identified QTL as QTL that are in complete population gametic phase disequilibrium

\footnotetext{
* Correspondence and reprints
}

E-mail: jdekkers@iastate.edu 
with the observed genetic polymorphism (i.e. the QTL itself or a tightly linked marker), in contrast to marked QTL, for which the observable polymorphism is in linkage equilibrium with the QTL.

The generally accepted strategy for using QTL information in selection, which will be referred to as standard QTL selection, is to select on a simple index of an estimate of the breeding value for the identified or marked QTL $(\alpha)$ and an estimated breeding value (EBV) of the residual polygenic effects of the individual: $I=\alpha+E B V[7,15]$. For additive QTL and when the components of the index are estimated using best linear unbiased prediction (BLUP) that includes the QTL as a fixed or random effect [8], standard QTL selection maximizes the response from the current to the next generation. Standard QTL selection, however, does not maximize the single generation response for QTL with non-additive effects [4], nor does it maximize the response over multiple generations, even for additive QTL $[9,11,14]$. Thus, the selection emphasis placed on the QTL in standard QTL selection, which is determined by the QTL breeding value, is not optimal for many situations.

The methods to optimize the use of a single identified QTL in selection were developed by Dekkers and van Arendonk [5] and Manfredi et al. [13]. Both methods focused on selection on a single identified QTL. Dekkers and Chakraborty [6] evaluated the benefit of optimal selection on a single identified QTL for a range of parameters and found substantial differences between optimal and standard QTL selection for QTL with overdominance.

With the advances in molecular genetics, multiple QTL are being identified for many livestock species [1] and, therefore, the information from more than one QTL must be incorporated in selection procedures. Standard QTL selection can accommodate multiple QTL by including the sum of breeding values of the individual QTL. Selection emphasis on an individual QTL is then determined by the relative magnitude of its breeding value. Hospital et al. [10] investigated QTL selection strategies in which equal emphasis was given to each QTL regardless of their effect, with the aim to fix all QTL as rapidly as possible. They, however, pointed out that it is unclear whether this strategy indeed minimizes the time to fixation and that optimal strategies for selection on multiple QTL are lacking.

In a companion paper, Chakraborty et al. [3] extended the method of Dekkers and van Arendonk [5] to allow optimization of selection on multiple QTL. The objectives of this paper were to implement this methodology for selection on two QTL and to evaluate the effect of linkage between QTL on characteristics of response to optimal QTL selection, in comparison to standard QTL and phenotypic selection. 


\section{METHODS}

\subsection{Population structure and genetic model}

The deterministic model described by Chakraborty et al. [3] was used to model selection on a trait affected by two identified QTL and residual polygenic effects in a population with discrete generations. Parameters for the population and genetic model were chosen to enable investigation and illustration of the properties of the optimal selection strategy and of the impact of linkage between QTL, rather than to be representative of practical breeding programs.

Selected fractions were $20 \%$ for both males and females. All candidates were genotyped for the identified QTL prior to selection and the effect and parental origin of each QTL allele was assumed known. Polygenic effects were modeled following the infinitesimal genetic model [2] but with constant variance. Both QTL were additive and had two alleles $\left(\mathrm{A}_{1}, \mathrm{~A}_{2}\right.$ and $\left.\mathrm{B}_{1}, \mathrm{~B}_{2}\right)$. Bilocus genotypes for the QTL are coded as, e.g., $\mathrm{A}_{1} \mathrm{~A}_{2} \mathrm{~B}_{1} \mathrm{~B}_{2}$, where the first allele for each locus designates the paternal allele. Starting frequencies of the favorable alleles $\left(\mathrm{A}_{1}\right.$ and $\left.\mathrm{B}_{1}\right)$ were 0.1 for both QTL and the starting population was in gametic phase equilibrium.

Allele substitution effects were equal to 1 and 1.5 in units of standard deviations $(\sigma)$ of polygenic EBV, which is the unit of interest when comparing alternative QTL selection strategies [5]. For a trait with heritability equal to 0.25 , these convert to allele substitution effects of 0.5 and 0.75 in units of standard deviations of true polygenic effects and to 0.25 and 0.375 in phenotypic standard deviation units if polygenic EBV are based on own phenotype only [6].

To evaluate the impact of linkage, recombination rates $(r)$ of 0.50 (unlinked), 0.20 , and 0.05 between the QTL were considered. A situation of a single QTL with an additive effect equal to the sum of the effects of the two QTL, i.e. $2.5 \sigma$, was also considered. Note that this is identical to a zero recombination rate between the QTL but with complete gametic phase disequilibrium in the base population, such that positive alleles are always in a coupling phase.

\subsection{Selection methods and objectives}

Optimal QTL selection was compared to standard QTL selection and to phenotypic selection. Selection was on the following general index [5]:

$$
I_{i j m t}=\theta_{m t}+\left(E B V_{i j m t}-\overline{B V}_{m t}\right)
$$

where $\theta_{m t}$ is the value assigned to individuals of the QTL genotype $m$ in generation $t$, which differed by selection strategy, and $E B V_{i j m t}$ is the polygenic EBV of animal $i$, which is deviated from the mean polygenic BV of individuals of genotype $m$ in generation $t\left(\overline{B V}_{m t}\right)$. Selection on index (1) was modeled by truncation selection across distributions of EBV by genotype, with means 
equal to $\theta_{m t}$ and standard deviation equal to $\sigma$. Given $\theta_{m t}$ and $\sigma$, proportions selected from each distribution were derived by bisection and were used to model changes in QTL frequencies and polygenic means, as described by Chakrabory et al. [3].

For phenotypic selection, means $\theta_{m t}$ were derived by regressing the mean phenotypic value of each genotype $\left(=q_{m}+\overline{B V}_{m t}\right.$ where $q_{m}$ is the sum of genotypic values for the QTL) towards an overall mean based on heritability [5, 6 , which was set equal to 0.25 . For standard QTL selection, means $\theta_{m t}$ were set equal to $\alpha_{m}+\overline{B V}_{m t}$, where $\alpha_{m}$ is the sum of breeding values for the two QTL based on allele substitution effects $[5,6]$.

The methodology developed by Chakraborty et al. [3] was used to derive optimal QTL selection strategies. Standard QTL selection provided the starting point for the iterative solution process. Optimal QTL selection strategies were obtained for two alternative objectives: (1) maximizing cumulative response after ten generations and (2) maximizing cumulative discounted response over ten generations. These will be referred to as optimal terminal and optimal discounted QTL selection, respectively. Cumulative discounted response was computed as $R=\sum_{t=0}^{T} w_{t} G_{t}$ where $G_{t}$ is the mean total genotypic value in generation $t$, including both the QTL and polygenes, and $w_{t}$ is a discount factor, which was computed based on an interest rate of $\rho=0.1$ per generation as: $w_{t}=1 /(1+\rho)^{t}$. Note that the terminal response is given by $G_{10}$.

The optimization program provides optimal fractions to select from each genotype in each generation. Based on the standard normal distribution theory, these fractions can be used to derive the standardized truncation point that is associated with genotype $m$ in generation $t, x_{m t}$. Following Dekkers and van Arendonk [5], differences in truncation points between genotypes were translated to the differences between means $\theta_{m t}$ that are assigned to each genotype in index (1) as:

$$
\theta_{m t}-\theta_{\text {ref }, t}=\left(x_{\text {ref }, t}-x_{m t}\right) \sigma
$$

where ref refers to an arbitrary reference genotype. Here, genotype $A_{1} A_{2} B_{1} B_{2}$ was used as the reference genotype. The means derived by (2) quantify the emphasis that is put on each QTL genotype in index (1) under optimal selection. These will be referred to as optimal genotype values, in contrast to standard genotypic values $\left(q_{m}\right)$, which refer to the impact of QTL genotype on phenotype.

Optimal genotype values computed by equation (2) apply to the bilocus QTL genotype. To quantify the emphasis that is placed on individual QTL, single locus genotype values were computed by averaging optimal bilocus genotype values over genotypes for the other locus. For example, the mean optimal genotype value for $A_{1} A_{1}$ was obtained as the average of the optimal bilocus genotype values for genotypes that included $A_{1} A_{1}$, weighted by the frequency of each genotype. 


\subsection{Gametic phase disequilibrium}

Selection generates gametic phase disequilibrium between QTL and between the QTL and polygenes [2] (gametic phase disequilibrium among polygenes was ignored by assuming constant polygenic variance). Denoting $P_{i j}$ as the frequency of QTL haplotype $i j$, and $P_{i}$ the frequency of allele $i$, gametic phase disequilibrium between the two QTL was computed following Lewontin [12] as:

$$
D^{\prime}=\left(P_{\mathrm{A}_{1} \mathrm{~B}_{1}} P_{\mathrm{A}_{2} \mathrm{~B}_{2}}-P_{\mathrm{A}_{1} \mathrm{~B}_{2}} P_{\mathrm{A}_{2} \mathrm{~B}_{1}}\right) / D_{\max }
$$

where

$$
D_{\max }=\min \left(P_{\mathrm{A}_{1}} P_{\mathrm{B}_{1}}, P_{\mathrm{A}_{2}} P_{\mathrm{B}_{2}}\right) \quad \text { when } D^{\prime}<0
$$

and

$$
D_{\max }=\min \left(P_{\mathrm{A}_{1}} P_{\mathrm{B}_{2}}, P_{\mathrm{A}_{2}} P_{\mathrm{B}_{1}}\right) \quad \text { when } D^{\prime}>0 .
$$

Gametic phase disequilibrium between the QTL and polygenes was quantified by the correlation of standard genotypic values for the QTL $\left(q_{m}\right)$ with polygenic breeding values as:

$$
r_{t}=\frac{\operatorname{cov}\left(q_{m}, \overline{B V}_{m t}\right)}{\sqrt{\operatorname{var}\left(q_{m}\right)} \sqrt{\operatorname{var}\left(\overline{B V}_{m t}\right)+\sigma_{\mathrm{pol}}^{2}}}
$$

where $\sigma_{\mathrm{pol}}^{2}$ is the polygenic variance within QTL genotype. Variances and covariances of $q_{m}$ and $B V_{m t}$ were computed based on haplotype means weighted by haplotype frequencies.

\section{RESULTS}

\subsection{Response to selection}

\subsubsection{Maximizing terminal response}

Cumulative responses over ten generations in population average QTL, polygenic, and total genotypic values are listed in Table I for the alternative selection strategies and genetic models. Polygenic responses were based on mean polygenic breeding values and QTL responses on mean genotypic values for the two QTL, both deviated from the genetic level in the starting population.

As expected, optimal terminal QTL selection resulted in the greatest mean total genotypic value in the final generation (Tab. I), followed by phenotypic selection and then standard QTL selection. The greater terminal response for phenotypic than standard QTL selection agrees with previous studies, which investigated selection on a single identified QTL $[9,11,14]$. The difference 
Table I. Terminal responses over ten generations (in phenotypic standard deviations) to phenotypic, standard QTL, and optimal QTL selection with one or two QTL with a varying recombination rate $(r)$. QTL substitution effects are 1.0 and 1.5 standard deviations of polygenic EBV for two QTL and 2.5 for one QTL. Polygenic heritability is 0.25 and starting frequencies of favorable alleles are equal to 0.1 for all QTL. In brackets, total responses are also expressed relative to response for phenotypic selection.

\begin{tabular}{|c|c|c|c|c|c|}
\hline \multirow{2}{*}{$\begin{array}{l}\text { Selection } \\
\text { strategy }\end{array}$} & \multirow{2}{*}{$\begin{array}{c}\text { Response } \\
\text { component }\end{array}$} & \multicolumn{3}{|c|}{ Two QTL } & \multirow[t]{2}{*}{ One QTL } \\
\hline & & $r=0.05$ & $r=0.20$ & $r=0.50$ & \\
\hline \multirow[t]{3}{*}{ Optimal } & Total & $4.31(103.8)$ & $4.27(102.6)$ & $4.27(102.4)$ & $4.44(101.7)$ \\
\hline & Polygenic & $3.20 \quad(97.3)$ & 3.18 (96.6) & 3.18 (96.6) & $3.32(102.1)$ \\
\hline & QTL & $1.11(128.7)$ & 1.09 (125.4) & 1.09 (123.7) & $1.12(100.3)$ \\
\hline \multirow[t]{3}{*}{ Standard } & Total & $3.97 \quad(95.5)$ & $3.97 \quad(95.5)$ & $3.98 \quad(95.5)$ & $4.10 \quad(93.9)$ \\
\hline & Polygenic & $2.84 \quad(86.4)$ & 2.85 (86.6) & 2.86 (86.9) & $2.98 \quad(91.7)$ \\
\hline & QTL & $1.12(130.4)$ & $1.12(128.9)$ & 1.12 (127.6) & $1.13(100.4)$ \\
\hline \multirow[t]{3}{*}{ Phenotypic } & Total & 4.15 & 4.16 & 4.17 & 4.37 \\
\hline & Polygenic & 3.29 & 3.29 & 3.29 & 3.25 \\
\hline & QTL & 0.86 & 0.87 & 0.88 & 1.12 \\
\hline
\end{tabular}

between phenotypic and standard QTL selection was, however, slightly less for two QTL than when their effects were concentrated into one QTL $(-4.5 \%$ for two QTL compared to $-6.1 \%$ for one QTL). Phenotypic selection resulted in a similar QTL response than standard QTL selection with a single QTL but in substantially less QTL response with two QTL. Lost polygenic response for standard QTL selection relative to phenotypic selection was, however, smaller for one QTL than for two QTL.

Extra terminal response from optimal over phenotypic selection was greatest for two tightly linked QTL $(r=0.05)(+3.8 \%)$ and smallest for the one QTL case $(+1.7 \%)$ (Tab. I). With two QTL, the greater QTL response was the main reason for the extra response from optimal over phenotypic selection; polygenic response was slightly lower for optimal than for phenotypic selection. This is in contrast to the one QTL case, where greater polygenic response was responsible for the greater total response for optimal over phenotypic selection and QTL responses were similar between the two strategies. Note that the one QTL cases is equivalent to the two QTL case with complete linkage and complete disequilibrium. Thus, the one QTL case is not an extension to complete linkage of the two QTL cases examined here, for which the QTL were in equilibrium in the base population.

Extra response from optimal over standard QTL selection increased with linkage from $+6.9 \%$ for unlinked loci to $+8.3 \%$ for tightly linked QTL (Tab. I). 
With one QTL, extra response from optimal selection was intermediate to these values at $+7.8 \%$. Extra polygenic response was the main reason for the difference in total response between optimal and standard QTL selection. This confirms the previous finding for the one QTL case by Dekkers and van Arendonk [5] that optimal terminal QTL selection maximizes polygenic response over generations while achieving near fixation of the QTL at the end of the planning horizon.

Tight linkage between the QTL resulted in slightly greater total response for optimal selection compared to moderate or no linkage (4.31 vs. 4.27, Tab. I). In contrast, linkage slightly reduced total response for standard QTL and phenotypic selection. Although differences were small, this illustrates the ability of the optimal strategy to capitalize on linkage. This will be further addressed later in the results section.

Trends in cumulative responses to selection are illustrated in Figure 1 for standard and optimal QTL selection for two unlinked (Fig. 1a) and tightly linked (Fig. 1b) QTL. Standard QTL selection reached the maximum QTL genotypic value after seven generations. Increases in QTL values were more gradual for optimal terminal QTL selection but also nearly reached their maximal value by generation ten. Linkage had limited effects on QTL responses. Because of greater emphasis on the QTL, standard QTL selection resulted in a lower polygenic response in the initial generations and in a lower cumulative polygenic response over the planning horizon. This is similar to the results described previously for a single QTL [5].

\subsubsection{Maximizing cumulative discounted response}

Comparisons between the three selection strategies when the objective was to maximize cumulative discounted response over ten generations are shown in Table II. As expected, optimal QTL selection resulted in the greatest response for all cases. However, in contrast to the terminal response, cumulative discounted responses were greater for standard QTL than for phenotypic selection when two QTL were present, because of greater QTL responses in early generations (Fig. 1). Standard QTL and phenotypic selection had similar cumulative discounted responses for the one QTL case, with lower responses for standard QTL selection in later generations offsetting early gains, and losses in discounted polygenic response offsetting gains in QTL discounted response.

The benefit of optimal over phenotypic selection was greater for cumulative discounted response (Tab. II) than for terminal response (Tab. I) and greater for two than for one QTL (Tab. II). The discounted polygenic response was lower for optimal QTL than for phenotypic selection but this was more than offset by the extra discounted QTL response. The extra cumulative discounted response from optimal over phenotypic selection was the greatest for tightly linked loci but did not increase consistently with degree of linkage. The impact 

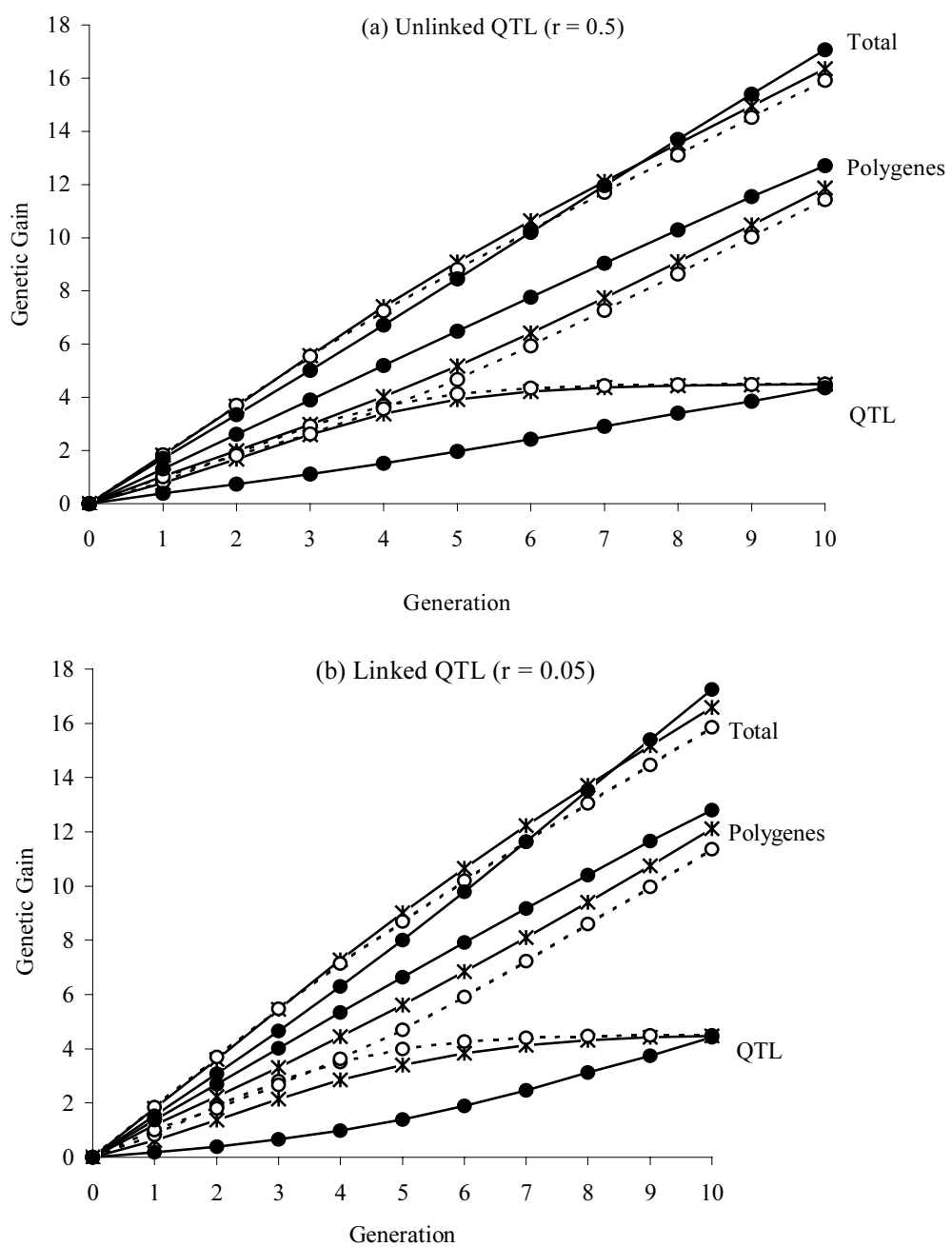

Figure 1. Cumulative gains in polygenic, QTL, and total genotypic means for standard (- - o - -), optimal terminal (-○-), and optimal cumulative discounted (一米-) selection for (a) two unlinked or (b) two tightly linked QTL $(r=0.05)$. Starting frequencies are 0.1 for both QTL.

of linkage on relative and absolute discounted total response was, however, small, with greater discounted polygenic response with tight linkage partially offset by lower discounted QTL response (Tab. II).

Extra response from optimal over standard QTL selection was smaller for cumulative discounted (Tab. II) than for terminal response (Tab. I) because of the emphasis on early gains in the former objective. Extra cumulative discounted response from optimal discounted over standard QTL selection was 
Table II. Cumulative discounted responses over ten generations (in phenotypic standard deviations) to selection with one or two QTL, with varying recombination rate $(r)$, for phenotypic, standard QTL, and optimal QTL selection, when the objective is to maximize cumulative discounted response. QTL substitution effects are 1.0 and 1.5 standard deviations of polygenic EBV for two QTL and 2.5 for one QTL. Polygenic heritability is 0.25 and starting frequencies of favorable alleles are equal to 0.1 for all QTL. Interest rate is $10 \%$ per generation. In brackets, total responses are also expressed relative to response for phenotypic selection.

\begin{tabular}{|c|c|c|c|c|c|}
\hline \multirow{2}{*}{$\begin{array}{l}\text { Selection } \\
\text { strategy }\end{array}$} & \multirow{2}{*}{$\begin{array}{l}\text { Response } \\
\text { component }\end{array}$} & \multicolumn{3}{|c|}{ Two QTL } & \multirow[t]{2}{*}{ One QTL } \\
\hline & & $r=0.05$ & $r=0.20$ & $r=0.50$ & \\
\hline \multirow[t]{3}{*}{ Optimal } & Total & $12.7(106.0)$ & $12.6(105.3)$ & $12.7(105.6)$ & $14.0(103.3)$ \\
\hline & Polygenic & $8.4 \quad(87.2)$ & $7.9 \quad(82.5)$ & $7.9 \quad(82.3)$ & (92.3) \\
\hline & QTL & $4.3 \quad(182.2)$ & $4.7 \quad(197.3)$ & $4.8 \quad(198.8)$ & $5.5 \quad(127.0)$ \\
\hline \multirow[t]{3}{*}{ Standard } & Total & $12.3(102.5)$ & $12.3(102.7)$ & $12.4(103.0)$ & $\begin{array}{ll}13.4 \quad(99.3) \\
\end{array}$ \\
\hline & Polygenic & $7.4 \quad(76.6)$ & $7.4 \quad(76.6)$ & $7.4 \quad(76.7)$ & (79.5) \\
\hline & QTL & $4.9 \quad(207.9)$ & $5.0 \quad(207.8)$ & $5.0 \quad(208.3)$ & $6.1 \quad(142.2)$ \\
\hline \multirow[t]{3}{*}{ Phenotypic } & Total & 12.0 & 12.0 & 12.0 & 13.5 \\
\hline & Polygenic & 9.6 & 9.6 & 9.6 & 9.2 \\
\hline & QTL & 2.4 & 2.4 & 2.4 & 4.3 \\
\hline
\end{tabular}

slightly greater for the one QTL case $(+4.0 \%)$ than for two tightly linked QTL $(+3.5)$, and the smallest $(+2.6 \%)$ for two QTL with moderate or no linkage. Optimal discounted QTL selection resulted in substantially greater discounted polygenic response than standard QTL selection, in particular for one QTL and for two tightly linked QTL, but this was partially offset by a lower discounted QTL response.

Figure 1 shows trends in response for optimal discounted QTL selection. Polygenic and QTL responses for selection that maximized cumulative discounted response were intermediate to those for standard QTL selection and to selection that optimized terminal responses, but tended to be closer to trends for the former. For both linked and unlinked QTL, optimal discounted QTL selection had similar total response as standard QTL selection in early generations but greater response in later generations. This was achieved by sacrificing some QTL response in early generations, which allowed an increase in polygenic response. As a result, optimal selection was able to maintain total response in early generations, while additional QTL response was capitalized on in later generations because the QTL were not yet fixed. The amount of QTL response sacrificed by optimal selection in early generations in favor of polygenic response was greater for tightly linked QTL than for unlinked QTL. 


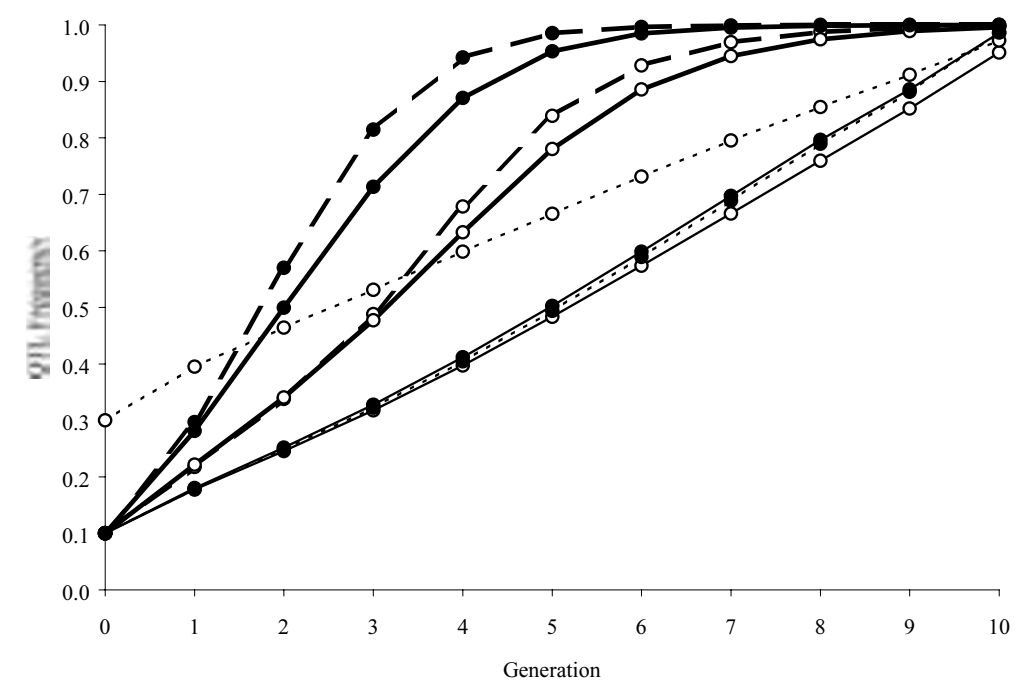

Figure 2. QTL frequencies for two unlinked QTL with effects equal to 1.0 (o) and 1.5 (•) standard deviations of polygenic EBV for standard (-- ), optimal cumulative discounted (—) and optimal terminal (— for 0.1 starting frequencies and - - - for starting frequencies of 0.3 and 0.1 ).

\subsection{QTL allele frequencies}

Figure 2 shows trends in frequencies for two unlinked QTL for standard and optimal QTL selection. Starting frequencies of the favorable QTL alleles were 0.1 . For optimal terminal QTL selection, an initial frequency of 0.3 for the smaller QTL (A) was also considered.

As expected, standard QTL selection resulted in rapid fixation of the favorable alleles, in particular for the QTL with larger effect. Similar trends, but with a lower rate of fixation, were observed for optimal QTL selection when the objective was to maximize cumulative discounted response.

For optimal QTL selection, trends in allele frequencies were nearly linear when the objective was to maximize terminal response, regardless of starting frequencies (Fig. 2). When starting frequencies were equal, trends were also similar for the two QTL, despite a difference in their genotypic effects. When starting frequencies were different, emphasis on each QTL was such that the frequencies increased at a nearly constant rate to reach near fixation at the end of the planning horizon. In contrast, with standard and optimal discounted QTL selection, rates of fixation were much greater for the QTL with the larger effect.

Figure 3 illustrates the effect of linkage on trends in QTL frequencies. Only results for tight linkage $(r=0.05)$ are shown; those for moderate linkage $(r=0.2)$ were intermediate. 


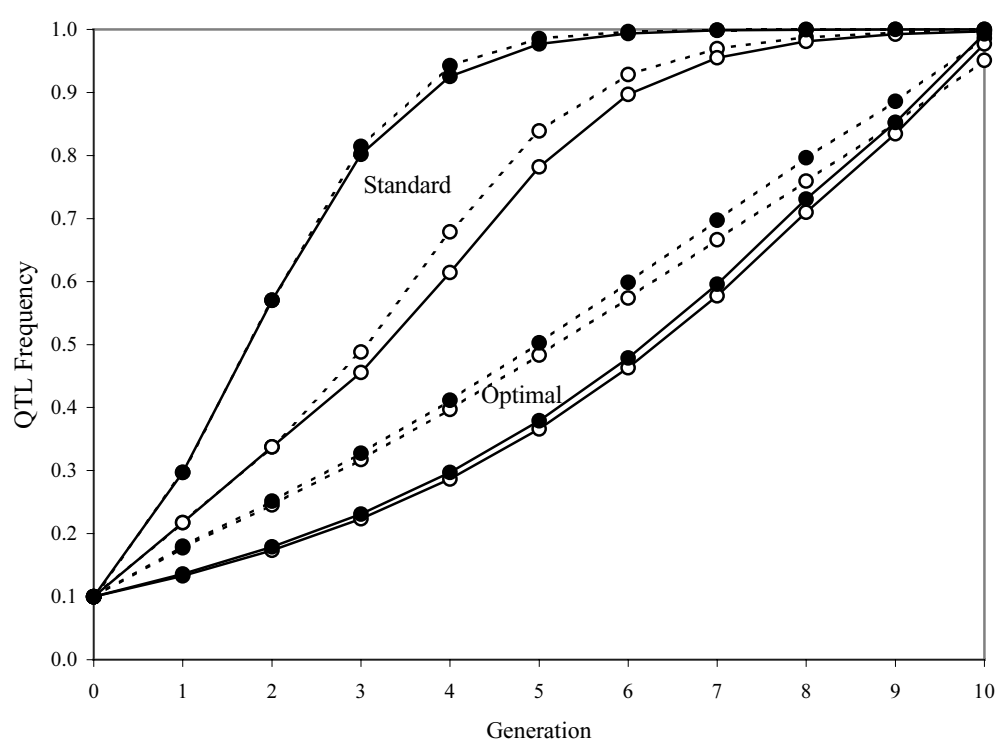

Figure 3. QTL frequencies for two unlinked (- - - ) or tightly linked $(r=0.05)$ $(-)$ QTL with effects equal to 1.0 (०) and $1.5(\bullet)$ standard deviations of polygenic EBV for standard and optimal terminal QTL selection. Starting frequencies are 0.1 for both QTL.

Linkage had a limited effect on QTL frequencies for standard selection (Fig. 3), although rates of fixation were slightly reduced with linkage because favorable alleles in repulsion phase were not released as quickly. For optimal selection, both QTL approached fixation regardless of the degree of linkage. However, increases in QTL frequencies were smaller with linkage than without linkage in early generations. The reason for the different effect of linkage for standard versus optimal QTL selection is explored in the following section.

\subsection{QTL haplotype frequencies}

Changes in frequencies for three QTL haplotypes are shown in Figure 4a for standard QTL selection and in Figure 4b for optimal terminal QTL selection. Standard QTL selection resulted in rapid fixation to a frequency of one of the favorable haplotype $\left(\mathrm{A}_{1} \mathrm{~B}_{1}\right)$ and to zero of the unfavorable haplotype $\left(A_{2} B_{2}\right)$. Standard QTL selection initially increased the frequency of the haplotype in the repulsion phase $\left(\mathrm{A}_{2} \mathrm{~B}_{1}\right)$ before reducing its frequencies to zero. Linkage had a limited impact on these trends, although the move to fixation of the favorable haplotype was slightly slower and the rise and subsequent decline of frequencies for repulsion phase haplotypes were slightly greater with linkage. This is caused by the slower break-up of linked QTL in repulsion phase. 

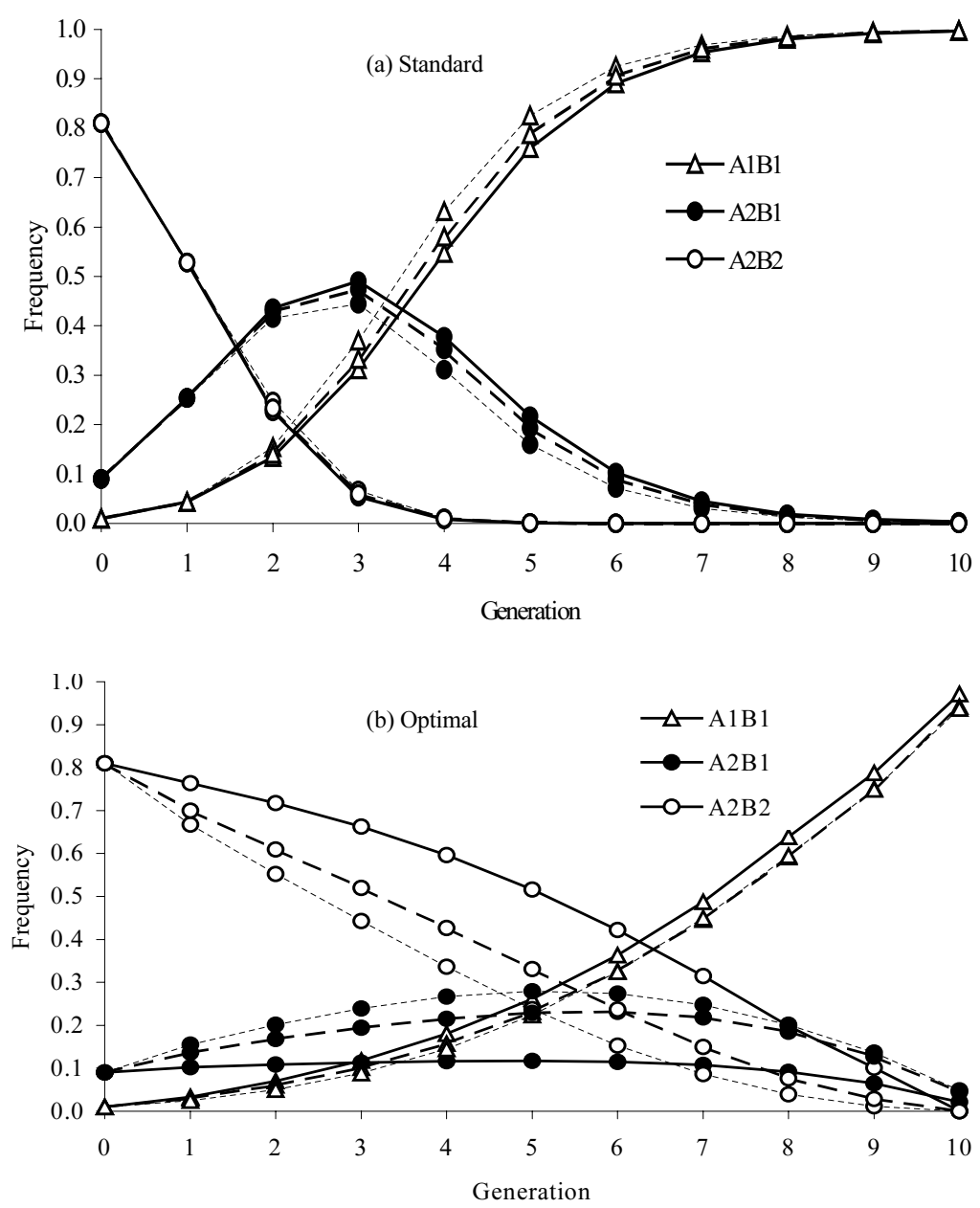

Figure 4. Frequencies of selected QTL haplotypes for two QTL with recombination rates equal to $0.5(---), 0.2(---)$, or 0.05 ( $(-)$, and effects equal to 1.0 (A) and 1.5 (B) standard deviations of polygenic EBV, for (a) standard and (b) optimal terminal QTL selection. Starting frequencies of the favorable alleles for each QTL $\left(\mathrm{A}_{1}\right.$ and $\left.\mathrm{B}_{1}\right)$ are 0.1 .

Quite different trends in haplotype frequencies were observed for optimal QTL selection (Fig. 4b). Frequencies of the favorable and unfavorable haplotypes also increased and decreased, respectively, but much more gradually than for standard QTL selection. Linkage had a limited impact on the increasing trend for the favorable haplotype but resulted in a slower decline in the frequency of the unfavorable haplotype. In contrast to standard QTL selection, frequencies for the repulsion phase haplotype were greatly impacted by linkage under optimal selection; without linkage, the frequency of the 
repulsion phase haplotype increased for the first five generations, after which it decreased towards zero. With tight linkage, however, the frequency of the repulsion phase haplotype was nearly constant for the first eight generations, followed by a gradual decline towards zero. With tight linkage, repulsion phase haplotypes do not break up through recombination and must be selected against in order to eventually fix the favorable QTL alleles. Thus, the initial increase in frequency of the repulsion phase haplotype with standard QTL selection (Fig. 4b) represents a wasted selection effort because it has to be selected against in later generations. This wastage is avoided by optimal QTL selection by minimizing the increase in frequency of such haplotypes. This ability to minimize wasted selection effort also explains why the benefit of optimal QTL over standard QTL and phenotypic selection is greater with tight linkage (Tab. I). Most of these effects disappeared, however, with moderate linkage, which behaved similar to unlinked QTL under optimal selection (Fig. 4b).

\subsection{Fractions selected}

The effect of linkage on selection strategies is further illustrated in Figure 5, which shows fractions selected from specific QTL genotypes with tight $(r=$ 0.05) and no linkage for standard (Fig. 5a) and optimal terminal QTL selection (Fig. 5b). The number of favorable and unfavorable haplotypes and alleles that is present in each genotype is also indicated. For brevity, only the results for generations 0,4 , and 8 are shown. The genotypes that are not depicted exhibited similar trends as the presented genotypes, based on the number of favorable and unfavorable haplotypes they contain.

For standard QTL selection, fractions selected from each genotype decreased with a declining QTL value of the genotype (Fig. 5a), as expected. The selected fractions decreased over generations because of the increasing frequency of the favorable alleles. Linkage had no impact on the fractions selected in the initial generation, as expected, and limited impact in later generations, which was primarily caused by differences in genotype frequencies.

For optimal QTL selection (Fig. 5b), differences in fractions selected between genotypes were not as large as for standard selection and linkage had a much greater impact. Without linkage, the selected fractions decreased with and were almost entirely determined by the number of favorable alleles, regardless of the linkage phase and the difference in the effect of the alleles between the two QTL. This is consistent with the observed similarity in frequency trends between the two QTL (Fig. 1). With tight linkage, however, the selected fractions were determined primarily by the number of favorable haplotypes, with a slight negative emphasis on the number of unfavorable haplotypes. For example, fractions selected were very similar for genotypes $A_{1} A_{1} B_{1} B_{2}$ ( 1 favorable and 0 unfavorable haplotypes) and $A_{1} A_{2} B_{1} B_{2}$ ( 1 favorable and 1 unfavorable haplotype). Genotypes with no favorable haplotypes were 


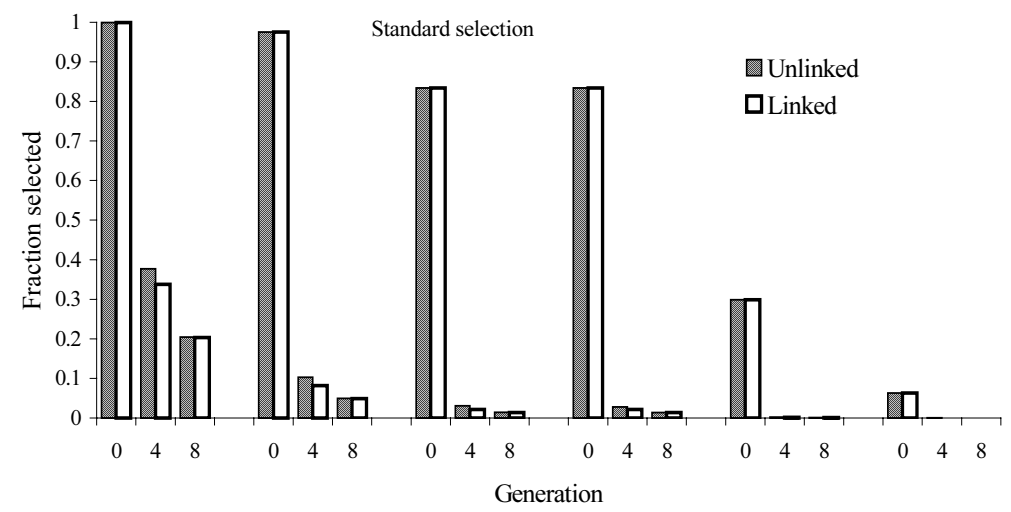

\begin{tabular}{|c|c|c|c|c|c|c|}
\hline Genotype & $\mathrm{A}_{1} \mathrm{~A}_{1} \mathrm{~B}_{1} \mathrm{~B}_{1}$ & $\mathrm{~A}_{1} \mathrm{~A}_{1} \mathrm{~B}_{1} \mathrm{~B}_{2}$ & $\mathrm{~A}_{1} \mathrm{~A}_{2} \mathrm{~B}_{1} \mathrm{~B}_{2}$ & $\mathrm{~A}_{1} \mathrm{~A}_{2} \mathrm{~B}_{2} \mathrm{~B}_{1}$ & $\mathrm{~A}_{1} \mathrm{~A}_{2} \mathrm{~B}_{2} \mathrm{~B}_{2}$ & $\mathrm{~A}_{2} \mathrm{~A}_{2} \mathrm{~B}_{2} \mathrm{~B}_{2}$ \\
\hline $\begin{array}{l}\text { No. favoral } \\
\text { haplotypes }\end{array}$ & 2 & 1 & 1 & 0 & 0 & 0 \\
\hline $\begin{array}{l}\text { No unfavor } \\
\text { haplotypes }\end{array}$ & able & 0 & 1 & 0 & 1 & 2 \\
\hline $\begin{array}{l}\text { No. favora } \\
\text { alleles }\end{array}$ & 4 & 3 & 2 & 2 & 1 & 0 \\
\hline
\end{tabular}

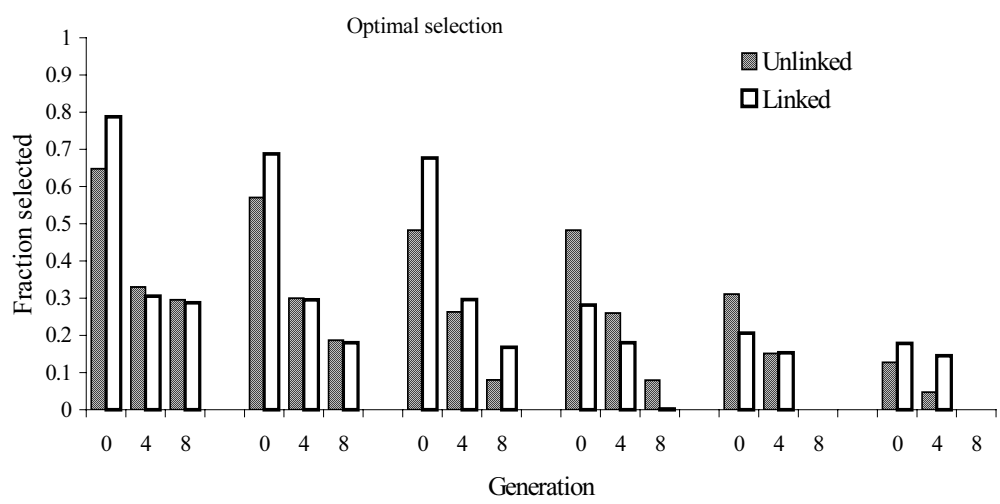

Figure 5. Proportions retained for breeding and haplotype characteristics for selected QTL genotypes in selected generations for standard and optimal terminal QTL selection for two unlinked ( $\square$ ) or linked ( $\square$ ) QTL, with effects equal to 1.0 (A) and 1.5 (B) standard deviations of polygenic EBV. Starting frequencies of the favorable alleles for each QTL $\left(\mathrm{A}_{1}\right.$ and $\left.\mathrm{B}_{1}\right)$ are 0.1 .

selected with the lowest emphasis; and, for those genotypes the fractions selected decreased slightly with increasing number of unfavorable haplotypes. With tight linkage, greater fractions of the most unfavorable genotype $A_{2} A_{2} B_{2} B_{2}$ were selected than without linkage. Consequently, the decline in frequency of the $A_{2} B_{2}$ haplotype observed with linkage in Figure $4 b$ was less rapid than without linkage. Moderate linkage ( $r=0.2$, results not shown) had results that were intermediate but closer to the situation with no linkage. 
Table III. Proportions selected and average genotypic values (in brackets) assigned to individual QTL genotypes under optimal QTL selection to maximize terminal response after ten generations for two unlinked $(r=0.5)$ or linked $(r=0.05)$ QTL with effects equal to $1.0(\mathrm{~A})$ and $1.5(\mathrm{~B})$ standard deviations of polygenic EBV. Starting frequencies of the favorable alleles for each QTL $\left(\mathrm{A}_{1}\right.$ and $\left.\mathrm{B}_{1}\right)$ are 0.1 .

\begin{tabular}{|c|c|c|c|c|c|c|}
\hline \multirow[b]{2}{*}{ Generation } & \multicolumn{6}{|c|}{ QTL genotype } \\
\hline & $\begin{array}{c}\mathrm{A}_{1} \mathrm{~A}_{1} \\
(+1.0) \\
\end{array}$ & $\begin{array}{c}\mathrm{B}_{1} \mathrm{~B}_{1} \\
(+1.5)\end{array}$ & $\begin{array}{c}\mathrm{A}_{1} \mathrm{~A}_{2} \\
(0)\end{array}$ & $\begin{array}{c}\mathrm{B}_{1} \mathrm{~B}_{2} \\
(0)\end{array}$ & $\begin{array}{c}\mathrm{A}_{2} \mathrm{~A}_{2} \\
(-1.0)\end{array}$ & $\begin{array}{c}\mathrm{B}_{2} \mathrm{~B}_{2} \\
(-1.5) \\
\end{array}$ \\
\hline & \multicolumn{6}{|c|}{ Unlinked QTL $(r=0.5)$} \\
\hline 1 & $\begin{array}{c}0.45 \\
(0.28)\end{array}$ & $\begin{array}{c}0.46 \\
(0.28)\end{array}$ & $\begin{array}{l}0.34 \\
(0)^{*}\end{array}$ & $\begin{array}{l}0.35 \\
(0)\end{array}$ & $\begin{array}{c}0.16 \\
(-0.60)\end{array}$ & $\begin{array}{c}0.16 \\
(-0.62)\end{array}$ \\
\hline 3 & $\begin{array}{c}0.28 \\
(0.09)\end{array}$ & $\begin{array}{c}0.28 \\
(0.09)\end{array}$ & $\begin{array}{l}0.25 \\
(0)\end{array}$ & $\begin{array}{c}0.25 \\
(0)\end{array}$ & $\begin{array}{c}0.16 \\
(-0.36)\end{array}$ & $\begin{array}{c}0.15 \\
(-0.37)\end{array}$ \\
\hline 5 & $\begin{array}{c}0.27 \\
(0.12)\end{array}$ & $\begin{array}{c}0.27 \\
(0.13)\end{array}$ & $\begin{array}{c}0.23 \\
(0)\end{array}$ & $\begin{array}{c}0.23 \\
(0)\end{array}$ & $\begin{array}{c}0.13 \\
(-0.41)\end{array}$ & $\begin{array}{c}0.13 \\
(-0.45)\end{array}$ \\
\hline 7 & $\begin{array}{c}0.25 \\
(0.18)\end{array}$ & $\begin{array}{c}0.25 \\
(0.20)\end{array}$ & $\begin{array}{l}0.2 \\
(0)\end{array}$ & $\begin{array}{l}0.2 \\
(0)\end{array}$ & $\begin{array}{c}0.09 \\
(-0.63)\end{array}$ & $\begin{array}{c}0.07 \\
(-0.75)\end{array}$ \\
\hline 9 & $\begin{array}{c}0.24 \\
(\mathrm{n} / \mathrm{a})^{* *}\end{array}$ & $\begin{array}{c}0.24 \\
(0.45)\end{array}$ & $\begin{array}{c}0.16 \\
(0)\end{array}$ & $\begin{array}{c}0.14 \\
(0)\end{array}$ & $\begin{array}{l}0.01 \\
(\mathrm{n} / \mathrm{a})\end{array}$ & $\begin{array}{c}0.0 \\
(\mathrm{n} / \mathrm{a})\end{array}$ \\
\hline & \multicolumn{6}{|c|}{ Linked QTL $(r=0.05)$} \\
\hline 1 & $\begin{array}{c}0.32 \\
(0.17)\end{array}$ & $\begin{array}{c}0.33 \\
(0.19)\end{array}$ & $\begin{array}{c}0.26 \\
(0)\end{array}$ & $\begin{array}{c}0.27 \\
(0)\end{array}$ & $\begin{array}{c}0.19 \\
(-0.23)\end{array}$ & $\begin{array}{c}0.18 \\
(-0.25)\end{array}$ \\
\hline 3 & $\begin{array}{c}0.29 \\
(0.12)\end{array}$ & $\begin{array}{c}0.29 \\
(0.12)\end{array}$ & $\begin{array}{l}0.25 \\
(0)\end{array}$ & $\begin{array}{c}0.25 \\
(0)\end{array}$ & $\begin{array}{c}0.17 \\
(-0.25)\end{array}$ & $\begin{array}{c}0.17 \\
(-0.25)\end{array}$ \\
\hline 5 & $\begin{array}{c}0.28 \\
(0.12)\end{array}$ & $\begin{array}{c}0.28 \\
(0.12)\end{array}$ & $\begin{array}{c}0.25 \\
(0)\end{array}$ & $\begin{array}{c}0.24 \\
(0)\end{array}$ & $\begin{array}{c}0.15 \\
(-0.33)\end{array}$ & $\begin{array}{c}0.15 \\
(-0.34)\end{array}$ \\
\hline 7 & $\begin{array}{c}0.28 \\
(0.17)\end{array}$ & $\begin{array}{c}0.27 \\
(0.17)\end{array}$ & $\begin{array}{c}0.23 \\
(0)\end{array}$ & $\begin{array}{c}0.23 \\
(0)\end{array}$ & $\begin{array}{c}0.1 \\
(-0.53)\end{array}$ & $\begin{array}{c}0.09 \\
(-0.56)\end{array}$ \\
\hline 9 & $\begin{array}{l}0.27 \\
(\mathrm{n} / \mathrm{a})\end{array}$ & $\begin{array}{l}0.26 \\
(\mathrm{n} / \mathrm{a})\end{array}$ & $\begin{array}{c}0.16 \\
(0)\end{array}$ & $\begin{array}{c}0.15 \\
(0)\end{array}$ & $\begin{array}{c}0.0 \\
(\mathrm{n} / \mathrm{a})\end{array}$ & $\begin{array}{c}0.0 \\
(\mathrm{n} / \mathrm{a})\end{array}$ \\
\hline
\end{tabular}

* Used as reference genotype.

** Could not be computed because selected proportion was zero for one of the genotypes.

Fractions selected from genotypes for each of the two QTL, averaged over the other QTL, are given in Table III for optimal terminal QTL selection for two unlinked or tightly linked QTL. The selected fractions were very similar for the two QTL, regardless of linkage, which is consistent with the similar trends in QTL frequencies observed in Figure 3. 


\subsection{Optimal QTL genotype values}

Table IV shows genotype values assigned to selected QTL genotypes under optimal terminal selection on unlinked QTL, which were derived following equation (2). Optimal genotype values generally declined with the number of favorable alleles. Double heterozygotes $\left(\mathrm{A}_{1} \mathrm{~A}_{2} \mathrm{~B}_{1} \mathrm{~B}_{2}\right)$ were, however, assigned a slightly greater value than the genotypes that were homozygous for alternate alleles (e.g. $\mathrm{A}_{1} \mathrm{~A}_{1} \mathrm{~B}_{2} \mathrm{~B}_{2}$ ), which is most likely associated with gametic phase disequilibrium among the QTL and between the QTL and polygenes.

Optimal single locus genotype values are given in Table III. Similar to proportions selected, optimal single locus genotype values were nearly equal for the two QTL, indicating that equal emphasis is placed on both loci. For both QTL the value of the heterozygote was closer to the value of the favorable homozygote. This was also observed by Dekkers and van Arendonk [5] for one QTL and relates to reducing gametic phase disequilibrium between the QTL and polygenes (see later).

If selection treats the two QTL loci as independent, the optimal genotype value of a bilocus QTL genotype will equal the sum of the optimal single locus genotype values. For example, the genotype value of genotype $A_{1} A_{2} B_{1} B_{2}$ will equal the single locus value for $A_{1} A_{2}$ plus the single locus value for $B_{1} B_{2}$. Comparing the optimal single locus genotype values of Table III to the bilocus genotype values of Table IV shows that this independence does not hold for optimal selection, even if the QTL are unlinked.

\subsection{Gametic phase disequilibrium}

The extent of gametic phase disequilibrium generated by optimal terminal and standard QTL selection between the two QTL is presented in Figure 6a for unlinked and tightly linked QTL. Standard QTL selection resulted in increasing negative disequilibrium between the two QTL over generations (Fig. 6a). Negative disequilibrium here indicates that individuals with a more favorable genotype for one QTL tended to have a more unfavorable genotype for the other QTL, which is caused by an excess frequency of repulsion phase haplotypes. Linkage increased the amount of disequilibrium for standard QTL selection because repulsion phase haplotypes recombined less frequently to break up the disequilibrium (Fig. 4a).

Disequilibrium between the QTL was small but negative for optimal selection without linkage (Fig. 6a). With tight linkage, however, optimal selection resulted in a large positive disequilibrium between the QTL. Positive disequilibrium indicates that individuals that had a favorable genotype for one QTL also tended to have a favorable genotype at the other QTL. The large positive disequilibrium for optimal selection resulted from its ability to maintain the frequency of repulsion phase haplotypes at low frequencies (Fig. 4b). 
Table IV. Optimal genotype values assigned to selected QTL genotypes under optimal selection on two unlinked QTL (A and B) with effects equal to 1.0 (A) and 1.5 (B) standard deviations of polygenic EBV, to maximize terminal response at the end of ten generations. Starting frequencies of the favorable alleles for each QTL $\left(\mathrm{A}_{1}\right.$ and $\left.\mathrm{B}_{1}\right)$ are 0.1 .

QTL genotype

(number of favorable alleles/effect of QTL genotype on phenotype)

\begin{tabular}{|c|c|c|c|c|c|c|c|c|c|c|}
\hline Generation & $\begin{array}{c}\mathrm{A}_{1} \mathrm{~A}_{1} * \\
\mathrm{~B}_{1} \mathrm{~B}_{1} \\
(4 /+2.5)\end{array}$ & $\begin{array}{c}\mathrm{A}_{1} \mathrm{~A}_{1} \\
\mathrm{~B}_{1} \mathrm{~B}_{2} \\
(3 /+1.0)\end{array}$ & $\begin{array}{c}\mathrm{A}_{1} \mathrm{~A}_{2} \\
\mathrm{~B}_{1} \mathrm{~B}_{1} \\
(3 /+1.5)\end{array}$ & $\begin{array}{l}\mathrm{A}_{1} \mathrm{~A}_{2} \\
\mathrm{~B}_{1} \mathrm{~B}_{2} \\
(2 / 0)\end{array}$ & $\begin{array}{l}\mathrm{A}_{1} \mathrm{~A}_{2} \\
\mathrm{~B}_{2} \mathrm{~B}_{1} \\
(2 / 0)\end{array}$ & $\begin{array}{c}\mathrm{A}_{1} \mathrm{~A}_{1} \\
\mathrm{~B}_{2} \mathrm{~B}_{2} \\
(2 /-0.5)\end{array}$ & $\begin{array}{c}\mathrm{A}_{2} \mathrm{~A}_{2} \\
\mathrm{~B}_{1} \mathrm{~B}_{1} \\
(2 /+0.5)\end{array}$ & $\begin{array}{c}\mathrm{A}_{2} \mathrm{~A}_{2} \\
\mathrm{~B}_{1} \mathrm{~B}_{2} \\
(1 /-1.0)\end{array}$ & $\begin{array}{c}\mathrm{A}_{1} \mathrm{~A}_{2} \\
\mathrm{~B}_{2} \mathrm{~B}_{2} \\
(1 /-1.5)\end{array}$ & $\begin{array}{c}\mathrm{A}_{2} \mathrm{~A}_{2} \\
\mathrm{~B}_{2} \mathrm{~B}_{2} \\
(0 /-2.5)\end{array}$ \\
\hline 1 & 0.42 & 0.22 & 0.23 & $0^{* *}$ & 0.00 & -0.16 & -0.14 & -0.43 & -0.45 & -1.10 \\
\hline 2 & 0.14 & 0.06 & 0.06 & 0 & -0.06 & -0.21 & -0.20 & -0.32 & -0.33 & -0.79 \\
\hline 3 & 0.12 & 0.06 & 0.05 & 0 & -0.03 & -0.18 & -0.17 & -0.30 & -0.31 & -0.77 \\
\hline 4 & 0.15 & 0.08 & 0.08 & 0 & -0.02 & -0.18 & -0.17 & -0.32 & -0.34 & -0.86 \\
\hline 5 & 0.19 & 0.11 & 0.11 & 0 & -0.01 & -0.19 & -0.17 & -0.37 & -0.40 & -1.04 \\
\hline 6 & 0.25 & 0.15 & 0.15 & 0 & 0.00 & -0.23 & -0.19 & -0.46 & -0.51 & -1.38 \\
\hline 7 & 0.34 & 0.20 & 0.21 & 0 & 0.00 & -0.32 & -0.24 & -0.64 & -0.75 & -2.16 \\
\hline 8 & 0.48 & 0.29 & 0.31 & 0 & 0.00 & -0.66 & -0.37 & -1.08 & -1.49 & $\mathrm{n} / \mathrm{a}^{* * *}$ \\
\hline 9 & 0.87 & 0.52 & 0.58 & 0 & 0.00 & $\mathrm{n} / \mathrm{a}$ & -0.79 & -2.32 & $\mathrm{n} / \mathrm{a}$ & $\mathrm{n} / \mathrm{a}$ \\
\hline 10 & 2.07 & 0.75 & 1.23 & 0 & -0.10 & -0.59 & 0.39 & -0.87 & -1.16 & $\mathrm{n} / \mathrm{a}$ \\
\hline
\end{tabular}

* The first allele refers to that inherited from the sire.

** Genotype $\mathrm{A}_{1} \mathrm{~A}_{2} \mathrm{~B}_{1} \mathrm{~B}_{2}$ was used as the reference genotype from which all other values were deviated.

*** Values could not be computed because fraction selected was zero for that genotype. 

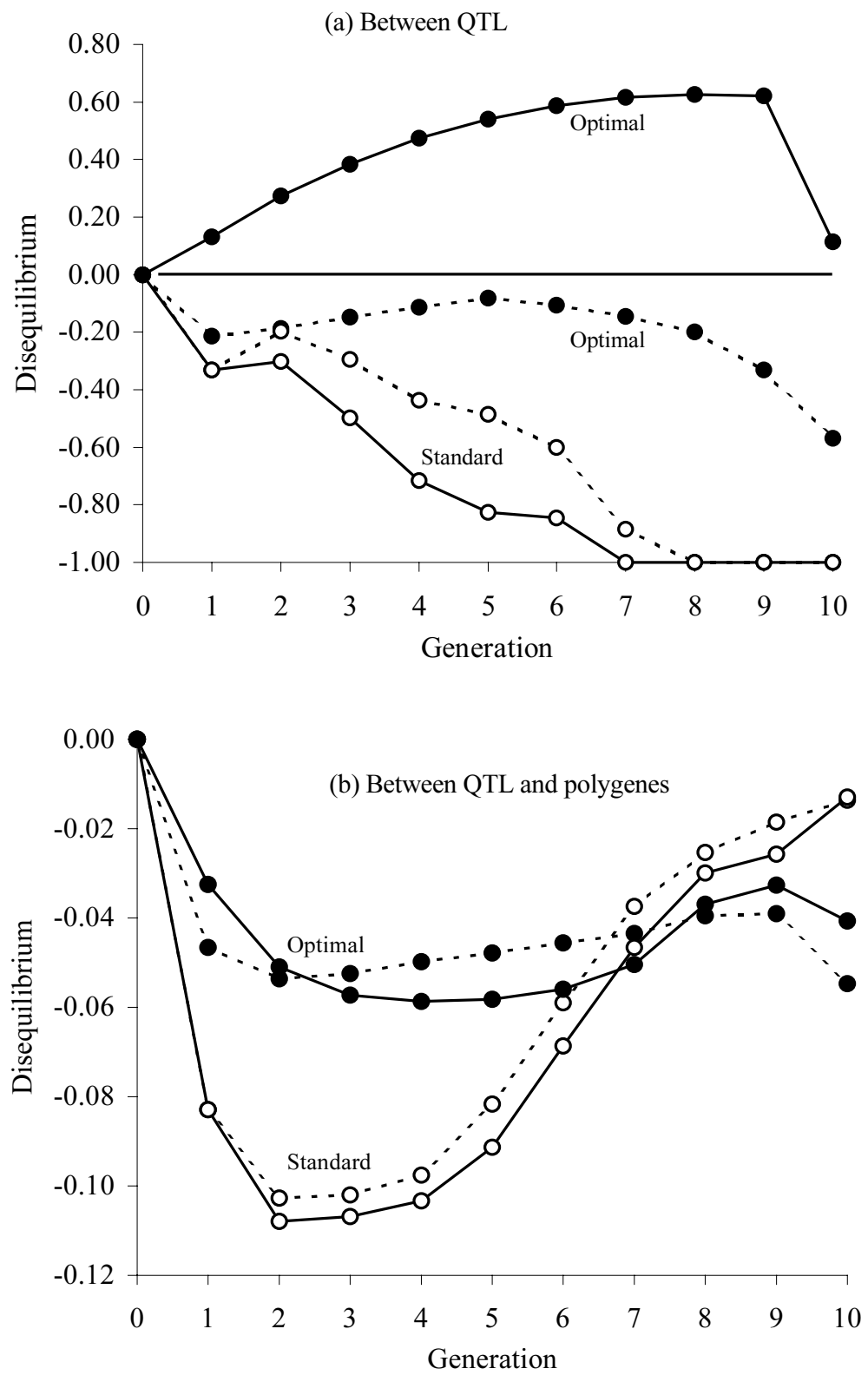

Figure 6. Disequilibrium between two unlinked (- - - ) or linked $(r=0.05)$ QTL $(-)$ QTL for standard (•) and optimal (o) QTL selection.

Both standard and optimal QTL selection resulted in negative disequilibrium between the QTL and polygenes for all cases (Fig. 6b), indicating that individuals with more favorable QTL genotypes tended to have lower 
polygenic breeding values. During the first five generations, correlations of QTL with polygenic values were twice as large for standard than for optimal QTL selection. In later generations the correlation reduced towards zero for standard QTL selection, as the QTL were moved to fixation. Tight linkage between the QTL slightly increased the extent of disequilibrium for standard QTL selection. For optimal selection, tight linkage resulted in slightly greater disequilibrium in early and late generations but in less disequilibrium in intermediate generations.

\section{DISCUSSION}

This study was focused on the evaluation of optimal strategies for simultaneous selection on two QTL, in comparison to standard QTL and phenotypic selection, and on the effect of linkage between the QTL on these strategies. Optimal strategies were derived using the methodology and algorithm developed by Chakraborty et al. [2].

Although only a limited number of scenarios was investigated, the results indicate that the benefit of optimal QTL selection over phenotypic selection is greater when the identified effects are distributed over multiple QTL. This holds for both terminal response (Tab. I) and for cumulative discounted response (Tab. II). Phenotypic selection has more difficulty in selecting for genes of small effect and, therefore, the benefit of being able to directly select on such genes is greater than for genes of large effect. The benefit of optimal over standard QTL selection was, however, greater for one QTL than for two QTL (Tabs. I and II), unless the QTL were tightly linked and the objective was to maximize cumulative discounted response (Tab. II). Tight linkage between the two QTL increased the advantage of optimal over standard QTL selection but moderate linkage $(r=0.2)$ had a limited impact and behaved very similar to selection on unlinked QTL.

Upon comparing optimal QTL selection with and without linkage between the two QTL, the results presented clearly demonstrate that the optimal strategy is able to take into account the ability for haplotypes that are in repulsion phase to recombine in future generations. This resulted in even greater response to selection with tight linkage than without linkage (Tab. I). This is in contrast to phenotypic and standard QTL selection, which wasted selection effort by selecting for haplotypes that are in repulsion phase in early generations. For these strategies, linkage resulted in slightly lower genetic gain, although the differences were not large. Most of these effects, however, disappeared with moderate linkage $(r=0.2)$, which behaved similar to selection on unlinked QTL because there is sufficient opportunity for QTL to recombine.

Only additive QTL were considered here, which is the most favorable situation for standard QTL selection. Greater differences between optimal 
and standard QTL selection are expected for QTL with dominance, as was demonstrated by Dekkers and Chakraborty [6] for the one QTL case. Epistasis between QTL may further increase the benefit of optimizing selection. Opportunities to capitalize on complex genetic inheritance, i.e. multiple QTL, linkage, epistasis, etc., will, however, be limited by the ability to accurately estimate associated parameters. In the present study, all parameters were assumed known without error. Estimation errors, which will be larger for complex genetic models, will limit the benefit of optimal selection. Nevertheless, these findings illustrate the potential of the optimization methods developed by Chakraborty et al. [3] to develop optimal selection strategies to capitalize on the complex genetic systems and interactions that are being uncovered through molecular genetic technology.

Several interesting features of optimal terminal selection were observed in Figures 2, 3, and 5 that deserve further discussion. First, both QTL reached near fixation by the end of the planning horizon (Fig. 2). Second, selection emphasis differed little between the two QTL when starting frequencies were equal, despite a difference in effects between QTL, as is evident from Figure 3 by the overlapping trends in QTL frequencies, and from Table III from the nearly equal fractions selected and equal optimal genotype effects for the two QTL. Third, trends in QTL frequencies were nearly linear when the QTL were unlinked, even when the two QTL started at different frequencies (Fig. 2).

To understand the impact of these findings it is beneficial to reformulate the selection objective of maximizing genetic level in the final generation to maximizing the polygenic value in the final generation subject to fixation of both QTL in the final generation. This equivalence of objectives holds for the QTL effects considered here and is based on the finding that in the optimal strategy both QTL were essentially fixed in the final generation. Thus, the assertion here is that the reformulated objective would lead to a similar selection strategy as the objective of maximizing terminal gain that was implemented here. From the reformulated objective, it is indeed evident that there is no advantage to putting more emphasis on the larger QTL if both start at the same frequency. After all, the selection effort that is required to move the favorable allele to fixation is equal for both QTL and does not depend on the effect of the QTL. The near linear trends that were observed with different starting frequencies (Fig. 2) further assert this principle. Thus, when the objective is to maximize terminal response, emphasis on a QTL is primarily determined by its starting frequency and not by the magnitude of its effect. It is important to note that the size of the QTL effects will be important when the objective is to maximize cumulative discounted response, as illustrated by the more rapid fixation of the QTL with larger effects (Fig. 2).

The latter also confirms the validity of the strategy of putting equal emphasis on each QTL when the objective is to reach fixation for all QTL as quickly as 
possible and all QTL start at the same frequency. This strategy was adopted in a simulation study by Hospital et al. [10], in which selection was exclusively on the QTL and all QTL had the same starting frequency. It is clear that also in that case the selection objective is not enhanced by giving QTL with greater genetic values more emphasis. Based on this argument, Hospital et al. [10] selected on the expected number of favorable QTL alleles contained by an individual. The results presented here indicate that this strategy would indeed be optimal for unlinked QTL because the selected fractions (Fig. 5) and optimal values for each genotype (Tab. IV) decreased with the number of favorable alleles. For tightly linked QTL, however, the optimal emphasis depended primarily on the number of favorable haplotypes, rather than on the number of favorable alleles, with some consideration of the number of unfavorable haplotypes (Fig. 5). Further work is needed to extend this result to multiple groups of linked QTL, which was the situation considered by Hospital et al. [10].

Hospital et al. [10] selected exclusively on markers with no consideration of other polygenic effects. Including residual polygenic effects in the selection decision requires consideration of not only the relative emphasis between QTL but also the emphasis on QTL versus polygenes. This was the situation considered here. The results presented in Tables III and IV for terminal selection on unlinked QTL show that, although the effects of the QTL on phenotype were additive and independent, the values assigned to the QTL under optimal selection behaved in a non-additive manner. Specifically, the average value assigned by the optimal strategy to the heterozygotes for each QTL was not central to that of the two homozygotes (Tab. III). In addition, when comparing optimal bilocus genotype values of Table IV to the optimal single locus genotype values of Table III, the optimal genotype values at the two QTL were not additive. Both types on non-additivity, as observed under optimal selection, are likely related to gametic phase disequilibrium among the QTL and between the QTL and polygenes. It is well known that selection on an aggregate objective induces negative associations between components of the aggregate (i.e. the two QTL and polygenes), which reduces response to selection in future generations [2]. Even for, unlinked loci, negative associations and their effects on response to selection will persist over multiple generations [2]. Optimal selection is able to anticipate the impact of negative associations on future responses to selection and, therefore, reduces the build-up of negative disequilibrium, as illustrated in Figures $6 \mathrm{a}$ and $6 \mathrm{~b}$. This is accomplished in part by a reducing emphasis on the QTL and in part by putting differential emphasis on alternate QTL genotypes. The latter results in the observed non-additivity of optimal QTL genotype values. It is not clear how important the assignment of non-additive effects under optimal selection is; responses may be robust to moderate changes in optimal QTL values. 


\section{ACKNOWLEDGEMENTS}

Financial support from the Pig Improvement Company and the NRI Competitive Grants Program/USDA (award no. 98-35205-6736) is greatly appreciated. This is Journal Paper No. J-19214 of the Iowa Agriculture and Home Economics Experiment Station, Ames, Iowa, USA (Project No. 3456) and supported by the Hatch Act and State of Iowa Funds. During her stay at ISU, L. Moreau was funded by INRA.

\section{REFERENCES}

[1] Andersson L., Genetic dissection of phenotypic diversity in farm animals, Nature Reviews, Genetics 2 (2001) 130-137.

[2] Bulmer M.G., The Mathematical Theory of Quantitative Genetics, Clarendon Press, Oxford, 1980.

[3] Chakraborty R., Moreau L., Dekkers J.C.M., A method to optimize selection on multiple identified quantitative trait loci, Genet. Sel. Evol. 34 (2002) 145-170.

[4] Dekkers J.C.M., Breeding values for identified quantitative trait loci under selection, Genet. Sel. Evol. 31 (1999) 421-436.

[5] Dekkers J.C.M., Van Arendonk J.A.M., Optimizing selection for quantitative traits with information on an identified locus in outbred populations, Genet. Research (1998) 257-275.

[6] Dekkers J.C.M., Chakraborty R., Potential gain from optimizing multi-generation selection on an identified quantitative trait locus, J. Anim. Sci. 79 (2001) 29752990.

[7] Falconer D.S., Mackay T.F.C., Introduction to Quantitative Genetics, Longman, Harlow, 1996.

[8] Fernando R.L., Grossman M., Marker-assisted selection using best linear unbiaised prediction, Genet. Sel. Evol. 21 (1989), 467-477.

[9] Gibson J.P., Short-term gain at the expense of long-term response with selection of identified loci, Proc. 5th World Cong. Genet. Appl. Livest. Prod. 21 (1994) 201-204.

[10] Hospital F., Goldringer I., Openshaw S., Efficient marker-based recurrent selection for multiple quantitative trait loci, Genet. Res., Camb. 75 (2000) 357-368.

[11] Larzul C., Manfredi E., Elsen J.M., Potential gain from including major gene information in breeding value estimation, Genet. Sel. Evol. 29 (1997) 161-184.

[12] Lewontin R.C., The interaction of selection and linkage. I. General considerations; heterotic models. Genetics 49 (1964) 458-472.

[13] Manfredi E., Barbieri M., Fournet F., Elsen J.M., A dynamic deterministic model to evaluate breeding strategies under mixed inheritance, Genet. Sel. Evol. 30 (1998) 127-148.

[14] Pong-Wong R., Woolliams J.A., Response to mass selection when an identified major gene is segregating, Genet. Sel. Evol. 30 (1998) 313-337.

[15] Soller M., The use of loci associated with quantitative effects in dairy cattle improvement, Anim. Prod. 27 (1978) 133-139. 